Mens

revue d'histoire intellectuelle de l'Amérique française

\title{
Les Québécois et leur héritage religieux
}

\section{Paul-Émile Roy}

Volume 2, numéro 1, automne 2001

URI : https://id.erudit.org/iderudit/1024456ar

DOI : https://doi.org/10.7202/1024456ar

Aller au sommaire du numéro

Éditeur(s)

Centre de recherche en civilisation canadienne-française

ISSN

1492-8647 (imprimé)

1927-9299 (numérique)

Découvrir la revue

Citer cet article

Roy, P.-É. (2001). Les Québécois et leur héritage religieux. Mens, 2(1), 17-33.

https://doi.org/10.7202/1024456ar d'utilisation que vous pouvez consulter en ligne.

https://apropos.erudit.org/fr/usagers/politique-dutilisation/ 


\section{ARTICLES \\ LES QUÉBÉCOIS \\ ET LEUR HÉRITAGE RELIGIEUX}

\section{PAUL-ÉMILE ROY \\ Écrivain}

Le Québec était autrefois une société catholique d'une grande homogénéité. Il formait une société cléricale que nos voisins appelaient «the priest-ridden province». En 1843, $\mathrm{M}^{\mathrm{gr}}$ Bourget, évêque de Montréal, écrivait : «Notre religion, c'est notre première distinction nationale, en même temps qu'elle est la base de nos institutions. C'est parce que nous sommes catholiques que nous sommes une nation en ce coin d'Amérique ${ }^{\prime} \gg$. Dans sa Brève histoire de l'Église catholique au Québec (1999), Lucia Ferretti affirmait: «De la Première Guerre mondiale jusqu'à la Révolution tranquille, l'Église se déploie pleinement comme organisatrice principale de la société québécoise²».

Jusque vers 1960 donc, le Québec était une société catholique. L'Église y était présente de façon très marquée par les nombreuses institutions qu'elle avait mises sur pied au cours des siècles précédents. La pratique religieuse était très répandue, et la très grande majorité des Québécois étaient considérés comme des croyants, et se disaient catholiques.

Or en l'espace de quelques décennies, de façon extrêmement rapide, le Québec s'est profondément transformé. On peut aujourd'hui parler, comme cela, sans scandaliser personne, d'un «Québec qui a rompu avec la religion traditionnelle ${ }^{3}$ ». Le 24 août 2001, Le Devoir titrait : «Une centaine d'églises montréalaises fermeront d'ici cinq ans.» Et le journaliste François Cardinal écrivait: «La ville aux cent clochers pourrait bientôt perdre son nom.» Fernand 
Dumont soulignait la rapidité avec laquelle la religion québécoise traditionnelle s'est effacée. «Ce qui me paraît véritablement étonnant, dit-il, c'est que tout ça s'est passé rapidement et sans crise religieuse, c'est-à-dire sans hérésie, sans sectes nouvelles, comme ça se produit habituellement ${ }^{4} . »$

Cette transformation rapide de la société québécoise est pour le moins étonnante et assez exceptionnelle. Elle explique peut-être certains malaises que nous ressentons actuellement, et le temps est venu d'essayer de comprendre ce qui s'est passé chez nous pendant la deuxième moitié du vingtième siècle. C'est même une tâche urgente et importante. Quelques auteurs ont abordé ce sujet, mais il me semble qu'on est loin de l'avoir épuisé, et c'est un champ de recherche presque inexploré qui s'offre à la curiosité des jeunes explorateurs! On a chanté le Québec, on l'a décrié; on l'a exalté, on l'a vilipendé. C'est ma conviction qu'on ne l'a pas beaucoup étudié, qu'on ne l'a pas analysé, qu'on ne l'a pas expliqué. Les images toutes faites, les tabous, les clichés tiennent lieu de réflexion. Nous avons délaissé les dogmes anciens, mais nous en avons inventé des nouveaux qui ne sont pas moins impérieux mais beaucoup plus gratuits. Tellement que plusieurs n'acceptent même pas d'en discuter et vouent aux gémonies ce qui ne concorde pas avec le discours convenu.

Les propos qui suivent n'ont rien de définitif. Je tente de décrire l'attitude des Québécois face à leur héritage religieux telle qu'elle s'est manifestée pendant la deuxième moitié du vingtième siècle. Mon idée centrale est que ce n'est pas une évolution que nous avons connue, que ce n'est pas une crise grave ou légère, mais une rupture avec un passé que nous tentions d'oublier, avec une identité que nous avons remisée dans les voûtes secrètes de l'histoire. Je voudrais essayer de décrire cette rupture, d'en chercher les raisons et d'en montrer si possible les conséquences.

Dans une entrevue qu'il accordait à Stéphane Stapinsky, Hubert Guindon disait de la Révolution tranquille : «Il y a eu une violence dans la rupture, une soudaineté, un radicalisme qui n'ont 
pas apporté et qui n'apportent rien de vraiment utile et de bienfaisant ${ }^{5} . \gg$ Ce radicalisme, si l'on en croit Fernand Dumont, a pris la forme d'un véritable procès du passé. «On a aboli, dit-il, les cours d'histoire et de géographie, tout en instaurant, de façon sousjacente, un immense procès de notre passé6.»

\section{Le procès de notre passé}

Ce procès a pris des formes multiples. Je pense, par exemple, à l'image de nos ancêtres que les «baby-boomers» ont transmise à leurs enfants. J'ai enseigné pendant de nombreuses années au cégep. Je recevais dans mes classes des jeunes garçons et des jeunes filles qui étaient les enfants des artisans de la Révolution tranquille. Or ces étudiants, en très grande majorité, avaient, de leurs grands-parents et de leurs aïeux, une image absolument méprisante. À les entendre, leurs grands-parents et leurs aïeux étaient tous des gens ignorants, tristes, soumis, "poignés», étroits d'esprit, fanatiques, intolérants, bigots, superstitieux. Une image qui ne coïncidait pas avec celle de mes parents que j'avais connus ni avec celle que nous ont transmise les écrits des conteurs et des voyageurs. Je voyais dans cette attitude de mes étudiants, une réaction profonde de refus de leur passé que leur avait infligée leurs parents et l'école qu'ils avaient fréquentée.

De même on présentait souvent nos ancêtres comme des gens timorés, repliés sur eux-mêmes, méfiants envers les étrangers. Or ce sont les Canadiens qui ont exploré tout l'intérieur du continent pendant que les Anglais se confinaient aux rivages de l'Atlantique. Ils se sont mêlés aux Amérindiens et ont participé étroitement à leur vie et à leurs activités. Après 1760 , ils ont joué un rôle important dans le développement des grandes compagnies anglaises qui ont développé le commerce des fourrures. A partir du XIX ${ }^{\mathrm{e}}$ siècle, le Québec a participé plus qu'aucun autre pays peut-être, au rayonnement missionnaire de l'Église sur tous les continents. Et même tout récemment, les mêmes gens qui accusaient les nationalistes québécois de se replier sur eux- 
mêmes, faisaient tout ce qu'ils pouvaient pour empêcher le Québec de participer aux organisations internationales.

Il faut rattacher à cette imagerie lugubre celle de notre jansénisme morbide, étouffant. Jean LeMoyne, dans Convergences, voyait dans le jansénisme une des composantes de la mentalité des Canadiens français. Nous avions hérité, paraît-il, du jansénisme français et même d'un dualisme sombre qui remontait à la lointaine Antiquité. Ces propos faisaient bien rire un de mes amis belges nouvellement arrivé au Québec qui affirmait n'avoir jamais rencontré un peuple si peu janséniste que les Canadiens français. Notre société n'était pas marquée par le puritanisme américain, par exemple, et la joie de vivre et de s'amuseréclatait continuellement dans les traditions et les moeurs des Canadiens français.

Dans cette vision biaisée et méprisante de la vie plus simple, plus pauvre, plus sobre peut-être, plus austère, différente en tout cas de leurs ancêtres, ce qui s'exprimait chez mes étudiants, c'était un refus de leur passé, d'une manière de vivre qu'ils jugeaient non seulement périmée, mais misérable. Eux ils avaient rompu avec cette détresse et entraient triomphants dans l'ère de toutes les libérations. Il faut rattacher à cette démarche d'émancipation, le mépris avec lequel on condamnait ce qu'on appelait l'ascèse judéo-chrétienne. Selon ces nouveaux Canadiens français, leurs ancêtres avaient cultivé jusqu' au masochisme le sens de l'effort, du renoncement, du sacrifice, du mépris de soi. C'est la religion catholique qu'ils jugeaient responsable de ces perversions, ignorant que l'ascèse s'est développée en Orient, dans la Grèce et la Rome anciennes avant même l'avènement du christianisme. Ce refus systématique et buté de l'ascèse «judéo-chrétienne» qui écartait du même coup le sens de l'effort, de la discipline, et de la maîtrise de soi, était lui aussi une forme de refus du passé, de la réalité d'hier, de ce qui avait précédé. Ou si l'on veut, on rejetait l'ascèse «judéo-chrétienne» parce qu'elle était un reliquat d'une autre époque, parce qu'elle appartenait à une culture qui était maintenant périmée.

Tout cela évidemment était lié au catholicisme traditionnel, qui apparaissait comme une structure anachronique qu'il fallait 
abandonner. Je ne me situe pas ici au plan de la foi ou de la religion comme telle, mais à celui de la culture informant toute une société. Le Québec d'autrefois était une société «catholique» au sens religieux du terme, mais aussi au sens qu'il avait des traditions, des us et coutumes, une histoire qui se rattachaient au monde catholique. Mon père, qui ne pratiquait pas, appartenait à ce monde catholique tout naturellement, comme d'autres étaient protestants ou musulmans, ou bouddhistes. Or voilà que tout à coup, en l'espace de quelques décennies, les Québécois ne se reconnaissent plus dans ces croyances, ces représentations, ces rites, ces coutumes qui jusquelà informaient leur existence. Quand on lit aujourd'hui les textes de cette époque, qu'on écoute les chansons ou qu'on regarde les pièces qu'on y présentait, on a l'impression que ces gens avaient le sentiment qu'ils repartaient à zéro, que rien de valable n'avait existé avant eux. On parlait de Révolution tranquille. Ces gens, comme tous les révolutionnaires, avaient la conviction de refonder le monde, au moins notre monde à nous, le Québec.

La réalité catholique qui avait été liée si étroitement à notre identité historique, était automatiquement perçue comme caduque. Et l'Église, qui était le moteur de cette réalité catholique, était particulièrement déclassée, perçue comme un véritable dinosaure, moins intéressant pourtant que les monstres historiques reconstitués par la science et l'imagination! Le procès du passé inclut le procès de l'Église qui est instruit de façon souvent cavalière au grand détriment de la vérité historique et surtout au détriment de l'intelligence des véritables enjeux de la société québécoise ${ }^{7}$. Le temps est venu, me semble-t-il, de porter sur notre société un regard critique plus objectif, moins agressif, j'allais dire moins masochiste.

\section{Le rôle de l'Église}

J'aborde ici le problème très délicat du débat autour du rôle de l'Église dans l'histoire du Québec. Un débat qui n'en est pas un en réalité, mais qui prend la forme de jugements catégoriques, évidents, qui ne se discutent même pas. On retrouve ce discours chez les humoristes, les chansonniers, dans les médias, dans la 
littérature. Il y a là un champ de recherche inépuisable pour les sociologues, les historiens.

Relevons une impression générale : l'Église était omniprésente, tâtillonne, elle contrôlait les consciences, elle était intolérante, étroite d'esprit. Elle interdisait la danse dans les paroisses, elle imposait un index sévère, elle se méfiait de la nouveauté, elle s'était approprié le champ de l'éducation et des soins de santé. Le clergé, les communautés religieuses faisaient peser sur la province une atmosphère religieuse étouffante qui paralysait le déploiement de la culture et de la liberté. Et l'on pourrait poursuivre indéfiniment cette énumération.

Évidemment, cette critique était fondée, et il fallait de toute évidence opérer un aggiornamento de l'Église et de la société québécoise. C'est une des inspirations fondamentales de la Révolution tranquille qui émanait d'ailleurs, pour une part, de l'Église elle-même. Bien avant 1960, les mouvements d'action catholique avaient éveillé l'esprit critique et la volonté de changement des Québécois ${ }^{8}$. L'Association catholique de la jeunesse canadienne-française, fondée par des clercs, avait aussi développé une nouvelle sensibilité culturelle et nationaliste. L'Église avait participé étroitement à la formation du mouvement coopératif et à la création et au développement des syndicats des cultivateurs. On assimile toujours l'Église à la stagnation de l'ordre établi, on oublie qu'elle a aussi été au Québec un facteur de changement. Raymond Lemieux et JeanPaul Montminy écrivaient : «Il y a indubitablement, au coeur même de la société traditionnelle, une créativité moderne du catholicisme québécois ${ }^{9} . »$

On reproche à l'Église d'avoir autrefois imposé une censure sévère dans le domaine des livres, du cinéma, des diverses publications. On dénonce des pratiques, et on a raison, mais on parle très peu des mesures adoptées par les gouvernants ou d'autres pouvoirs constitués. On dénonce les abus d'autorité des évêques au XIX ${ }^{e}$ siècle, et on parle très peu des menaces du 
gouverneur, de ses traitements tyranniques envers les journalistes du Canadien. Il démolit même l'imprimerie du journal, et son directeur, Étienne Parent, un modéré, passe cinq mois en prison pour avoir écrit des choses trop précises ${ }^{10}$. Je veux bien qu'on parle de la censure cléricale dans la société traditionnelle. Je voudrais aussi qu'on dénonce les autres formes de censure qui existaient, de façon à ne pas nous accabler de façon excessive. J'aimerais aussi qu'on n'oublie pas que la censure n'est pas disparue, et qu'elle est plus insidieuse que jamais.

La critique la plus sévère que les champions de la modernité adressent à l'Église québécoise, c'est d'avoir pactisé avec les conquérants, d'avoir établi «le pacte du trône et de l'autel». La question est très complexe, et sans vouloir excuser qui que ce soit, il faut essayer de comprendre le contexte de l'époque. On a bien l'impression, en lisant les enseignements ou les déclarations des évêques des premières décennies du régime anglais, qu'ils nourrissaient pour les institutions britanniques, une très forte considération. Or, il est bon de le noter, ils n'étaient pas les seuls à partager une telle admiration. En 1823, Le Canadien se félicite de ne pas dépendre des pays de la Sainte-Alliance européenne, mais de dépendre de l'Angleterre. «Bénissons le ciel, écrit le journal, de nous avoir fait naître dans un empire où l'on peut dire hautement tout ce qui est juste. Nous sommes nés sur le sol de la liberté, nous à qui la liberté a été si libéralement donnée, payons le plus vif intérêt à ceux que l'on va voir bientôt se battre pour la conserver ${ }^{11}$.»Yvan Lamonde écrit un peu plus loin : «Papineau, Parent et Garneau partagent un même britannisme, une même admiration pour la Constitution anglaise et les droits et libertés des sujets anglais ${ }^{12} . »$ Voici une déclaration qui a été faite en 1820 : «Rappelons-nous que sous le gouvernement français (arbitraire et oppresseur, à l'intérieur comme au dehors) les intérêts de cette colonie avaient été plus souvent négligés et mal administrés que dans aucune autre partie de ses dépendances [...]. Mais voyez le changement. George III, un souverain révéré pour son caractère moral, l'application à ses devoirs royaux et l'amour de ses sujets, succède à Louis $\mathrm{XV}$, un prince méprisé à bon droit 
pour ses débauches, son indifférence envers les besoins du peuple, ses prodigalités à même le trésor public, au bénéfice de ses favoris et de ses maîtresses. De ce jour, le règne de la loi remplace celui de la violence ${ }^{13}$.» Est-ce l'évêque de Québec qui parle? Non, c'est Papineau!

Les jugements catégoriques que l'on porte sur le servilisme des évêques canadiens au lendemain de la conquête sont donc à nuancer. L'admiration des institutions britanniques n'était pas le fait des seuls évêques. Il était très répandu chez plusieurs journalistes et plusieurs politiciens canadiens, ce qui est d'ailleurs assez surprenant quand l'on considère les abus du gouverneur et du conseil exécutif qui allaient entraîner la rébellion de 1837-1838, pas seulement dans le Bas-Canada, mais aussi dans le Haut-Canada. Mais pour ce qui est des évêques, il faut bien comprendre la situation dans laquelle ils se trouvaient au lendemain du Traité de Paris. L'ancienne colonie de la France était décapitée. Les cadres, les commerçants, les bourgeois étaient retournés en France. Les conquérants étaient les maîtres de cette société. «Dans une petite société ayant perdu ses élites, écrit Fernand Dumont, l'Église a alors assumé toutes les fonctions sociales essentielles» ${ }^{14}$.Très tôt, les évêques vont se révéler non seulement des hommes d'Église, mais les véritables chefs de cette communauté de Canadiens. Il va leur falloir jouer d'astuce. S'il y a chez le clergé de l'époque une réaction de rejet de l'évolution de la société française au temps de la Révolution et de Napoléon, il faut lui reconnaître aussi une bonne dose d'opportunisme et de sens politique. Les évêques se comportent comme les véritables chefs de la nation canadienne qu'ils protègent contre la volonté d'anglicisation et de protestantisation des conquérants. C'est ce qui explique le rôle hypertrophié que le clergé est appelé à jouer à la fin du dix-huitième siècle et au début du dix-neuvième. Ce phénomène d'ailleurs, n'est pas exclusif à la société canadienne. Dans toutes les sociétés colonisées, il se produit un phénomène analogue, comme l'ont noté bien des analystes.

Les événements de 1837-1838 vont créer une nouvelle conjoncture pour le clergé et pour l'Église. Les évêques interviennent 
contre la rébellion parce qu'ils savent qu'elle est suicidaire, comme bien des politiciens d'ailleurs, les Canadiens n'étant pas préparés pour vaincre l'armée britannique. Leur attitude est aussi motivée par le respect de l'autorité établie, mais ils prendront vite conscience, avec l'avènement de l'Union, que l'autorité «venant de Dieu» ne respectait pas toujours les droits humains. Au moment, donc, de l'Union, l'Église réagit contre le libéralisme mais aussi contre la volonté d'assimilation du nouveau régime politique, et va continuer à s'affirmer, sous un mode nouveau, comme le véritable chef de la société catholique canadienne. L'alliance d'hier entre le trône et l'autel étant affaiblie, c'est du côté de Rome qu'elle cherche un appui, et l'ultramontanisme prendra un grand essor. S'il a été si florissant chez nous, comme l'a expliqué Fernand Dumont ${ }^{15}$, c'est qu'il répondait en quelque sorte à une situation historique bien caractérisée.

Quand on considère cette période de notre histoire qui va de 1760 à 1900, on doit admettre que l'Église, comme les Canadiens eux-mêmes, se trouvait dans un contexte très difficile qu'on ne peut réduire à un schéma simpliste. Il faut se garder des caricatures partisanes. Il est obligatoire de donner de la perspective à nos analyses. Admettons que les évêques ont manqué de grandeur et de dignité, qu'ils étaient obsédés par l'idée que tout pouvoir vient de Dieu - notons en passant qu'on peut soutenir que l'autorité vient de Dieu à travers le peuple, tout aussi bien qu'à travers un roi. Mais ce qui est plus fondamental, c'est qu'ils n'ont pas compris que l'évolution du monde allait de la royauté à la démocratie. Il n'était pas facile, d'ailleurs, de se reconnaître, dans ce brassage des civilisations qui s'effectuait en Europe au dix-huitième et au dixneuvième siècles, et dont les effets se répercutaient fortement dans toute l'Amérique. Par la force des choses, nos évêques étaient d'une certaine façon restés des hommes d'ancien régime, et n'avaient pas compris qu'il fallait reconnaître l'autonomie des deux ordres, le temporel et le spirituel, le politique et le religieux, l'État et l'Église.

Le conflit entre conservatisme et libéralisme, entre le clergé et les intellectuels libéraux, entre l'Institut canadien et $\mathrm{M}^{\mathrm{gr}}$ Bourget, est un épisode de cette lutte de l'Église et de la société québécoise 
pour sortir d'une société de type d'ancien régime. L'évêque de Montréal défendait une théocratie catholique moyennageuse qui sera au vingtième siècle remplacée par la reconnaissance de l'autonomie des deux ordres : temporel et spirituel, qui s'exprimait à la fin du $\mathrm{XIX}^{\mathrm{e}}$ siècle par l'abandon par le pape des États pontificaux.

\section{La Révolution tranquille}

C'est ce débat déchirant que nous avons vécu en même temps que nous étions conquis et soumis à une puissance étrangère dotée d'une autre langue et d'une autre religion. Le temps allait maintenant venir où après de longues périodes de luttes nous allions tenter de liquider ce passé de frustrations, d'échecs, d'atermoiements, de temporisation, de soumission, en nous prenant en main et en assumant enfin notre destin. C'est le sens fondamental de ce que nous avons appelé la Révolution tranquille. C'était une opération nécessaire, emballante, dans laquelle il fallait éviter la précipitation et le fourvoiement.

Malheureusement, les révolutions se font rarement dans la sérénité. Même la nôtre, qui s'est faite sans violence, sans résistance, qui était «tranquille», a pris l'allure d'un raz-de-marée qui emportait tout le monde.

On peut dire que pour la première fois, la nation canadiennefrançaise se donnait un État à elle. Il fallait dès lors mettre fin à l'amalgame Église-société civile qu' avait provoqué notre situation de peuple conquis privé d'élite administrative, économique, culturelle. Très tôt cette volonté s'est concrétisée par la nationalisation de l'électricité, la création d'un ministère de l'Éducation, la prise en main des affaires sociales par le gouvernement, etc. Nous avions beaucoup de retards à rattraper, et la modernité nous poussait dans le dos.

On peut comprendre jusqu'à un certain point la précipitation aveugle avec laquelle nous avons mené l'opération. Il y avait tellement longtemps que la situation stagnait, depuis les luttes stériles 
du Parti patriote pour faire respecter les normes d'une saine administration, luttes qui devaient s'avérer un échec et se solder par l'imposition du régime de l'Union dont les intentions de minorisation et d'assimilation des Canadiens français étaient patentes. Malgré les apparences, la Confédération ne mettrait pas fin à l'Union mais en relancerait le mouvement. De 1867 à 1960, le gouvernement du Québec ne se comporterait pas comme celui d'un État, mais comme celui d'une province minoritaire qui devait se contenter des conditions que l'État central voulait bien lui concéder. C'est contre cet état de sujétion que les artisans de la Révolution tranquille s'élevaient avec fermeté, ce que traduisaient bien les slogans du jour : «Il faut que ça change», «Maîtres chez nous», «Égalité ou indépendance». Dans ce processus aussi impitoyable que nécessaire, le risque était de céder à l'emballement, de poursuivre les mirages de la nouveauté pour la nouveauté, de dilapider l'héritage au profit des satisfactions passagères.

En ce qui regarde l'Église, par exemple, il est clair qu'il fallait sortir de cette situation de crise dans laquelle pendant deux siècles elle avait en quelque sorte assumé des fonctions qui auraient dû normalement revenir au pouvoir civil. Il fallait mettre fin à ces fonctions de suppléance, ce que l'Église comprenait très bien, et ce dont elle prit elle-même l'initiative, ce qui explique que la révolution-le mot est un peu fort -s'est faite chez nous sans violence.

Mais ce n'est pas seulement l'Église comme institution qui était concernée par la Révolution tranquille. Le catholicisme, ce n'est pas seulement le clergé, c'est la religion du peuple, ses croyances, ses coutumes, sa mentalité. Le catholicisme québécois, ce n'est pas seulement ni surtout les évêques, les curés, ce sont ces petites gens qui allaient à l'église, vivaient dans la paroisse, avaient assimilé les grandes sagesses, les grandes vérités du christianisme, de l'Évangile. Que le curé fût éloquent ou pas, tous les dimanches, ils se réunissaient à l'Église, entendaient la lecture de l'Évangile qui leur enseignait à pardonner, à respecter les autres, etc. La vie de tout peuple a une dimension religieuse. Tout peuple fait l'expérience comme peuple d'une relation particulière à un sol, à la vie, au destin. Pensons aux 
Autochtones, aux pays asiatiques. Bali est un pays bouddhiste, la Thaillande, le Japon, la Chine, le Tibet se distinguent par des caractéristiques indéfinissables, des goûts, des convictions. Il y a dans tout peuple quelque chose d'irréductible à l'économie, à l'industrie, aux événements historiques. La religion fait partie de l'identité d'un peuple qu'on peut définir comme une communauté qui a une histoire commune, une certaine culture commune, une expérience commune de la transcendance.

Dans le contexte de notre situation de peuple colonisé, non seulement le clergé était amené à jouer un rôle disproportionné, mais la religion populaire elle-même, étant un élément de notre identité par opposition à la religion du conquérant qui tentait de la dominer, devenait en quelque sorte le refuge de toutes les valeurs humaines et nationales. Ce phénomène a été observé ailleurs par les analystes qui ont étudié les peuples colonisés. Les ressources nationales sont alors mobilisées, pour assurer la survivance, par la religion qui assume en quelque sorte le destin collectif. C'est ce qu'a montré Lanternari, par exemple, pour les Indiens d'Amérique. L'assimilation forcée entraîne la menace de la désagrégation de la culture et de la société indienne. «La religion médico-magique du peyolt, en s'adaptant aux nouvelles existences culturelles, et pour affronter la catastrophe qui s'abat sur les communautés, se constitue désormais comme religion de salut» ${ }^{16}$. De même Jacques Berque a montré que la religion devient le refuge des valeurs nationales du peuple colonisé ${ }^{17}$. Pierre Lefebvre a bien expliqué lui aussi ce qui arrive de la religion dans ce contexte ${ }^{18}$. Dans l'Irlande persécutée, explique Camille Bourniquel, le catholicisme s'est constitué en force de résistance, il a été «un noyau de cohésion : on ne saurait s'étonner que cette maintenance ait entraîné quelques ambiguïtés dans les rapports avec l'État et maintenu un conservatisme ${ }^{19} .{ }^{\prime}$

Au moment où un peuple ne se considère plus comme un peuple colonisé, au moment où il se prend en main, où il s'affirme, il fait éclater cet amalgame dans lequel il s'était réfugié 
pour parer les coups du destin. C'est cette opération que veut réaliser la Révolution tranquille. Le peuple québécois tente alors de renouveler la perception qu'il a de lui-même et des autres. Il s'engage dans une nouvelle existence qui prend la forme d'une libération.

Dans un processus comme celui-là, il y a risque que la confusion entraîne des conséquences désastreuses, que les véritables préjudices soient mal identifiés, et qu'en voulant corriger la situation, on se fourvoie dans des démarches aberrantes. Le sens de la Révolution tranquille était de sortir d'une situation aliénante, de nous libérer des effets nocifs d'une longue lutte historique pour nous retrouver nous-mêmes. Or, pour une part importante, cette Révolution a été vécue comme un refus de nous-mêmes, de notre histoire, de ce que nous avions été jusque-là. Tout ce qui nous touchait était mauvais. Notre histoire était honteuse. Mieux valait l'oublier. Je me rappelle cet étudiant de cégep qui m'expliquait que notre histoire n'était qu'un récit de défaites, qu'elle n'était pas intéressante, et qu'il valait mieux l'oublier. Notre héritage religieux nous rappelait un passé peu glorieux de frustrations et de morosité. Il fallait nous en détourner.

L'attitude que nous avons alors adoptée par rapport à la religion catholique a été désastreuse, non seulement pour la religion mais pour la culture, pour notre propre épanouissement. Toute notre vie, nos valeurs, notre culture, notre religion étaient liées au catholicisme. Il constituait, comme nous l'avons noté, l'élément capital de notre identité. «L'Église catholique, écrit Edmond de Nevers, est restée pour les Canadiens français, le centre d'union, de cohésion par excellence ${ }^{20}$. En renonçant au catholicisme comme nous l'avons fait, en le détruisant dans l'estime des gens, en l'avilissant de mille façons, nous avons laissé le Québécois nu, désarmé, honteux de lui-même, privé de sagesse, démuni, livré à toutes les modes et à toutes les doctrines même les plus gratuites et les plus délétères. C'est là qu'il faut chercher, pour une part, la cause de l'acuité particulière de la crise de l'éducation chez nous, de l'éclatement de la famille, du refus des valeurs morales. Je sais bien que l'Occident 
entier connaît une crise qui ressemble à la nôtre, mais elle prend chez nous des proportions et une gravité tout à fait exceptionnelles.

\section{Nous réapproprier notre héritage}

Le temps est venu, il me semble, pour les Québécois, de s'accepter tels qu'ils sont. Il ne s'agit pas de fermer les yeux sur nos travers, nos défauts, nos faiblesses. Je voudrais seulement que nous essayions d'être justes envers nous-mêmes, que nous cessions de nous flageller. Fernand Dumont affirmait que le grand problème de la culture contemporaine était de se donner «une conscience historique». Il se demandait si l'humanité était capable de «réactualiser son héritage», c'est-à-dire «ce qu'elle $\mathrm{es}^{\mathrm{t}^{21}}{ }^{\prime}$. Il disait qu'on ne crée pas les valeurs et, qu'en les vivant, on les modifie. La foi que nous pouvons avoir en l'homme ne s'invente pas, elle est un héritage que nous pouvons accueillir et enrichir ${ }^{22}$. Il disait même que «l'avenir se bâtit en faisant appel à ce qu'il y a de vivant dans le passé23.»

$\mathrm{Si}$, comme l'affirme Fernand Dumont, la culture moderne a besoin de se donner «une conscience historique», à plus forte raison une société comme le Québec, qui est secouée par des bouleversements très rapides, qui cherche à reformuler ou à se réapproprier son identité, a-t-elle besoin d'inventorier toutes ses ressources, d'assumer son histoire, de développer une véritable conscience historique.

Avoir une conscience historique, cela signifie se percevoir dans une continuité, dans un développement, dans un projet. Notre existence surgit du passé et s'ouvre sur l'avenir. Le présent est le produit pour une part d'un long cheminement. Mais l'homme d'aujourd'hui a perdu conscience de cette dimension de son existence. Il vit dans l'immédiat. Il a perdu le sens de la continuité. Il perçoit très difficilement ce qui se préparait dans le passé et se manifeste dans le présent. Son regard est narcissique. Il se projette dans son regard au lieu d'accueillir la réalité qu'il regarde. Quand il considère le passé, il y projette son 
propre malaise. Il l'aborde avec les critères d'aujourd'hui et s'en interdit l'intelligence. L'homme d'aujourd'hui vit dans le fonctionnel, l'unidimensionnel. Il est mal disposé à comprendre l'homme d'autrefois qui vivait une existence accordée aux rythmes de la nature, qui faisait l'expérience d'une relation aux autres et au monde ouverte sur la transcendance. Parce que l'homme d'aujourd'hui n'a pas accès à ce type d'expérience, il la voit comme une superstructure encombrante.

Tout cela étant dit, je me trompe peut-être, mais j'ai l'impression que nous tentons enfin de surmonter le traumatisme de la rupture, que nous tentons timidement de nous réapproprier l'héritage que nous avions délaissé. J'espère seulement ne pas m'illusionner. Il me semble que nous commençons à être plus capables d'examiner notre passé sans nous sentir obligés de le stigmatiser. Plusieurs ouvrages regardent l'histoire du Québec avec sérénité et sympathie, tentent de comprendre et d'expliquer ce qui s'est passé au lieu de juger et de condamner. «Le mythe de la grande noirceur ecclésiastique, écrivent Raymond Lemieux et Jean-Paul Montminy, tient sans doute beaucoup moins au vécu des ancêtres qu'au regard condescendant qu'une certaine intelligentsia, urbaine et scolarisée de très fraîche date, a porté sur eux dans la deuxième moitié du siècle» ${ }^{24}$. Le petit livre de Lucia Ferretti, que j'ai déjà cité, pourra aussi aider les Québécois à se donner une vue plus juste de leur passé. Dans un tout autre régistre, le charmant récit de Monique Boucher-Pratte, La Mémoire heureuse ${ }^{25}$, témoigne que le passé récent du Québec n'a pas été un enfer pour tout le monde.

Parvenu au terme de cet article, je me rappelle que Nietzche disait que la marque de la liberté, c'est de n'avoir plus honte de soi.

\section{NOTES}

' Cité par Fernand Dumont, Genèse de la société québécoise, Montréal, Boréal, 1993, p. 227.

${ }^{2}$ Lucia Ferretti, Brève histoire de l'Église catholique au Québec, 
Montréal, Boréal, 1999, p. 113.

${ }^{3}$ Jean-Claude Leclerc, «Une église catholique peut-elle accueillir une célébration non religieuse?», Le Devoir, 6 août, 2001, p. B6.

${ }^{4}$ Fernand Dumont, Un témoin de l'homme, Montréal, L'Hexagone, 2000 , p. 247. Entretiens colligés et présentés par Serge Cantin.

5 «L'insoumission sociologique, rencontre avec Hubert Guindon», Les Cahiers d'histoire du Québec au XXe siècle, n 8, automne 1997, p. 203. Propos recueillis par Stéphane Stapinsky.

${ }^{6}$ Fernand Dumont, Un témoin de l'homme, p. 289.

${ }^{7}$ Sur l'Église comme bouc émissaire, voir le Rapport Dumont, L'Église du Québec, un projet, Montréal, Fides, 1971, p. 71.

${ }^{8}$ Voir André-J. Bélanger, Ruptures et constantes. Quatre idéologies du Québec en éclatement, Montréal, HMH, 1978.

${ }^{9}$ Raymond Lemieux et Jean-Paul Montminy, Le Catholicisme québécois, Sainte-Foy, Les Éditions de l'IQRC, 2000, p. 48.

11) Voir Yvan Lamonde, Histoire sociale des idées au Québec, Montréal, Fides, 2000, p. 267.

"Ibid., p.205.

${ }^{12}$ Ibid., p. 209.

${ }^{13}$ Ibid., p. 210.

${ }^{14}$ Fernand Dumont, Pour la conversion de la pensée chrétienne, Montréal, HMH, Coll. Constantes, 1964, p. 158.

${ }^{15}$ Ibid., p. 21.

${ }^{16}$ Vittorio Lanternari, Mouvements religieux des peuples opprimés, Paris, François Maspéro, 1962, p. 118.

17 Jacques Berque, Dépossession du monde, Paris, Éd. Du Seuil, 1964, p. 11.

${ }^{18}$ Pierre Lefebvre, «Croyances et révolution», Parti pris, avril 1965, p. 11.

${ }^{19}$ Camille Bourniquel, «L'Irlande ou la réalité du mythe», Esprit, avril 1965, p. 631.

2) Edmond de Nevers, L'Avenir du peuple canadien-français, Montréal, Fides, 1964, p. 71.

${ }^{21}$ Fernand Dumont, Un témoin de l'homme, p. 110.

${ }^{22}$ Ibid., p 90.

${ }^{23}$ Ibid., p. 203.

${ }^{24}$ Raymond Lemieux, Jean-Paul Montminy, op. cit., p. 48.

${ }^{25}$ Monique Boucher-Pratte, La Mémoire heureuse. Lumières 
personnelles sur la Grande Noirceur, Sillery, Les Éditions du Septentrion, 2000. 